\title{
OPTIMIZATION OF TRIO-CAVITY RAMAN LASER PUMPED BY PULSED GAUSSIAN BEAM
}

\author{
DINH XUAN KHOA AND CHU VAN LANH \\ Vinh University
}

\begin{abstract}
In this paper the set of dimensionless rate equations for the pump, Stokes and antiStokes powers in the trio-cavity Raman laser pumped by pulsed Gaussian beam is introduced. By numerical Runger-Kuta method, those equations are resolved and the optimal normalized parameters for enhancing the output powers are found out.
\end{abstract}

\section{INTRODUCTION}

Among the last previous works the CW Raman laser is the most interested [1-9]. The investigation for Raman lasers operating in pulse regime or pumped by pulse is still too little [10], specially, for Raman laser generating at anti-Stokes wave.

To make rich knowledge of Raman laser, in this paper we introduce a set of dimensionless rate equations to describe revolution of intra-cavity powers (in Sec. 2), which is used to find out the optimal principle parameters of laser pumped by pulsed Gaussian pulse to enhance the output power of anti-Stokes wave (in Sec. 3).

\section{DIMENSIONLESS RATE EQUATIONS WITH GAUSSIAN PULSE}

Consider a Raman laser operating in trio-cavity resonator, which is illustrated in Fig.1 and pumped by an external Gaussian pulse given by [11]:

$$
P_{e p}(t)=\frac{W}{\tau} \sqrt{\frac{\ln 2}{\pi}} \exp \left[-\left(\frac{\sqrt{\ln 2} t}{\tau}\right)^{2}\right],
$$

where $W$ and $\tau$ are the total energy and duration of the Gaussian pulse, respectively.

Now, we make symbolization as follows:

$$
\begin{gathered}
\gamma_{p} \approx \gamma_{s}=\xi_{l o s s 1} \gamma_{a}=\xi_{l o s s 2} \gamma_{e p}=\gamma ; \\
x=t / \gamma ; \frac{P_{p} W}{\tau}=Y_{1} ; \frac{P_{s} W}{\tau}=Y_{2} ; \frac{P_{a} W}{\tau}=Y_{3} ; \\
\frac{k_{p}+k_{s}}{k_{p}+k_{a}}=Q_{s a} ; G(\delta) \frac{8 \omega_{p} \mu_{0}}{\omega_{s}^{\pi} b}=Q ; \\
\frac{Q W k_{p} \omega_{s}}{k_{s} \gamma \tau}=\alpha_{1} ; \frac{Q k_{p} Q_{s a} \omega_{a}}{k_{s} \gamma \tau}=\alpha_{2} ; \frac{Q W \omega_{s}}{\gamma \tau}=\alpha_{3} ; \\
\frac{Q C W \omega_{a}}{\gamma \tau}=\alpha_{4} ; \frac{Q C W \omega_{s}}{\gamma \tau}=\alpha_{5} ; \frac{Q \xi_{s a} W \omega_{a}}{\gamma \tau}=\alpha_{6},
\end{gathered}
$$


where $\xi_{\text {loss } 1}$ and $\xi_{l o s s 2}$ are defined as the ratio of Stokes wave loss to that of anti-Stokes wave one and of Stokes wave to that of external wave one, respectively, $\gamma_{q}=\left(c / n_{q} L\right) \sqrt{R_{1 q} R_{2 q}}$ is the intra-cavity lifetime of photon q-mode or q-mode loss, $\gamma_{e p}=\left(c / n_{p} L\right) \sqrt{T_{1 q}}$ is the loss of external field, $n_{q}$ is the refractive index of q-field, $\omega_{q}$ is the frequency of q-mode, $R$ is the cavity mirror's reflectance, $T$ is the transmittance, the subscript " 1 " presents the front mirror that couples the external field, and "2" means back mirror, $G(\delta)$ is the Raman gain coefficient, coupling constant corresponding to third-order susceptibility $\chi^{(3)}$ of Raman medium [12], $\delta$ is the two-photon detuning for $1 \rightarrow 2$ transition (see Fig. 1), $C=\left[\left(k_{p}+k_{s}\right) / \Sigma k\right] \sin (\Delta l L / 2 / \Delta k L / 2)$ is the coupling coefficient corresponding to phase mismatch in four wave interaction, $\Sigma k=2 k_{p}+k_{s}+k_{a}, \Delta k=2 k_{p}-k_{s}-k_{a}$ is the phase mismatch, $L$ is the cavity length, $b$ is the confocal parameter, $\xi_{s a}=b_{s} / b_{a} \approx b_{p} / b_{a}=b / b_{a}$ is defined as the relation coefficient between confocal parameters of Stokes $\left(b_{s}\right)$ and antiStokes $\left(b_{a}\right)$ waves. Substituting (1), (2) and (3) into set of equations for intra-cavity powers [12] we obtain the dimensionless equations

$$
\begin{aligned}
& \dot{Y}_{1}(t)=-Y_{1}(t)-\alpha_{1} Y_{1}(t) Y_{2}(t)+\alpha_{2} Y_{1}(t) Y_{3}(t) \\
& +\frac{1}{\gamma_{\text {loss } 2}} \sqrt{Y_{1}(t)} \sqrt[4]{\frac{\ln 2}{\pi}} \sqrt{\exp \left(-\left(\frac{\sqrt{\ln 2} t}{\tau}\right)^{2}\right)} \\
& \left.\dot{Y}_{2}(t)=-Y_{2}(t)+\alpha_{3} Y_{1}(t) Y_{2}(t)-\alpha_{4} Y_{1}(t) Y_{3}^{(} t\right) \\
& \left.\dot{Y}_{3}(t)=-\frac{1}{\gamma_{l o s s 1}} Y_{3}(t)+\alpha_{5} Y_{1}(t) Y_{2}(t)-\alpha_{6} Y_{1}(t) Y_{3}^{(} t\right) \\
& \overline{\overline{\overline{ }}} \\
& \text { J }
\end{aligned}
$$

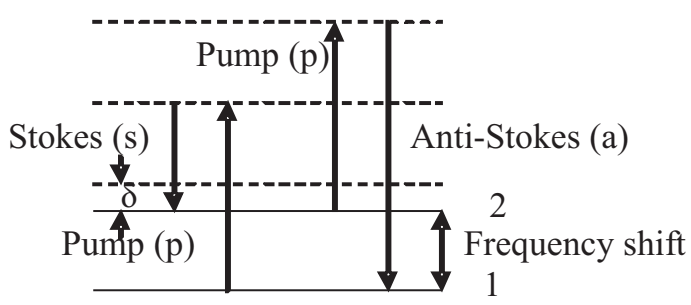

Fig. 1. Energy level diagram of the far-off resonance Raman process.

\section{OPTIMIZATION OF TRIO-CAVITY RAMAN LASER PUMPED BY PULSED GAUSSIAN BEAM}

As a sample for our investigation, the pump pulse, resonant cavity and Raman medium are chosen with parameters given following. The pump pulse at wavelength $1.06 \mu \mathrm{m}$ (of Diode-pumped Neodym laser for example) has an energy $W=\left(0 \div 4.5 \times 10^{-5}\right) \mathrm{J}$ and half duration time $\tau=10 \mathrm{ps}$, which is focused in center of laser cavity with beam waist 
to be $w=(0.05 \div 0.45) \mathrm{mm}$. The properties of resonant cavity are chosen as: reflectance coefficients $R_{1 p}=0.5, R_{2 p}=0.999, R_{1 s}=0.999, R_{2 s}=0.95, R_{1 a}=0.999, R_{2 a}=0.95$ (in this case $\left.\xi_{\text {loss }}=1\right)$, and length $L=(200 \div 1000) \mu \mathrm{m}$. The Raman medium is a sample of $D_{2}$-gas-in-glass fiber [4-6] with $\alpha(\delta) \approx 1.5 \times 10^{-9} \mathrm{~cm}^{2} / W$ [4], so $G(\delta)$ is calculated to be $\approx 1.3 \times 10^{-4} \mathrm{~cm}^{2} / W$. Consequently, the Raman wavelengths are $1.55 \mu \mathrm{m}$ for Stokes wave and $0.57 \mu \mathrm{m}$ for anti-Stokes one [5,6]. Using above given parameters to (10), and by numerical four-order Runger-Kuta method, we have obtained the following results.

\section{III.1. Influence of cavity length}

One of properties is cavity length. It influences not only on intra-cavity loss of waves, consequently on peak of pulses, but also on beam structure (beam waist). In Fig.2 there are dependence of Stokes power (a) and anti-Stokes power (b) on cavity length. One can see that: 1) the threshold value of cavity length is about $0.2 \mathrm{~mm} ; 2$ ) there is an optimal value of cavity length, which is to be $0.5 \mathrm{~mm}$ in this case; 3) changing of power for Stokes and anti-Stokes waves are similar.

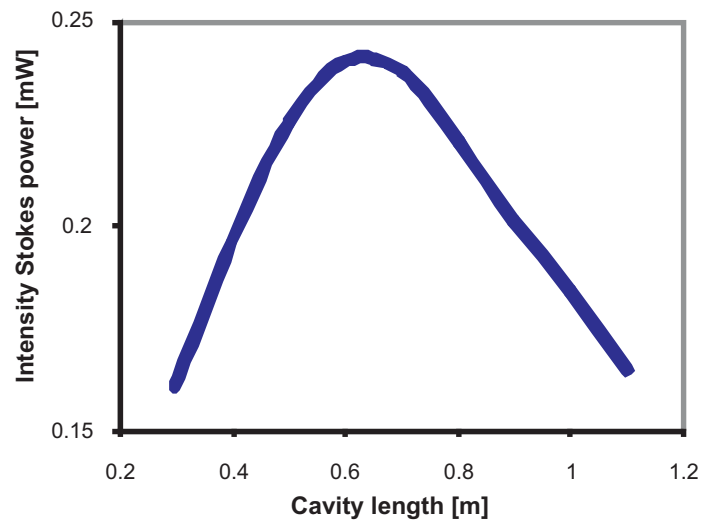

(a)

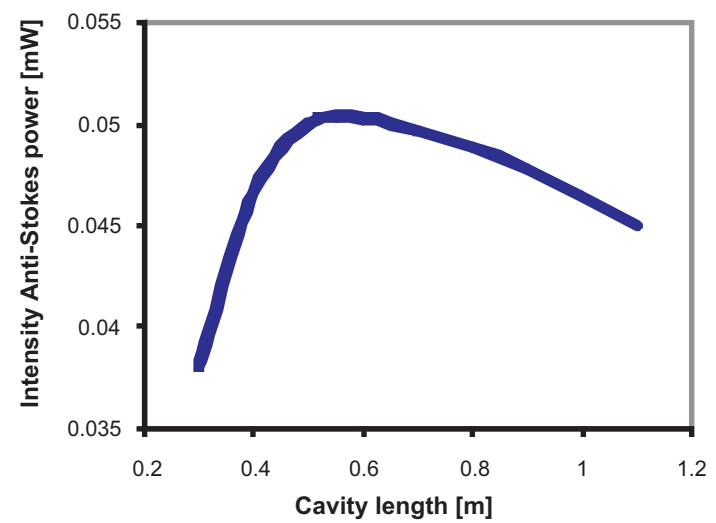

(b)

Fig. 2. Peak power vs cavity length. a) Stokes wave. b) Anti-Stokes wave.

As that proposed above, the length of cavity is equal to the length of active medium (Raman fiber), so at the short length, the Raman gain is low, i.e. the laser gain is not enough to exceed the loss, and consequently, the laser is not able to generate. The laser genetates when the laser gain reaches the threshold value corresponding to the threshold length. When the length increases, so the Raman gain increases and the generated power increases. After reaching the optimal power with the optimal length, since that the decreasing speed of the intra-cavity life time of the photon is more than that of the Raman gain when the length increases, so the intra-cavity power is decreases

\section{III.2. Influence of beam waist}

With given wavelengths, the confocal parameter will be changed with the beam waist. In nonlinear interaction the confocal parameter plays an important role, which influences on conversion efficiency [6]. 


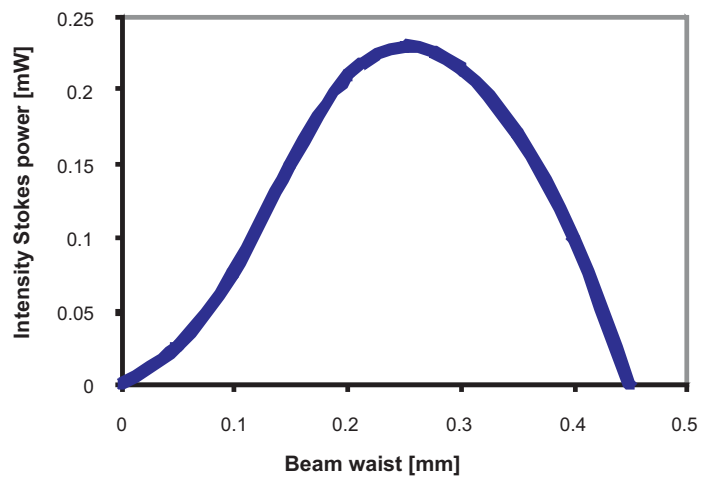

(a)

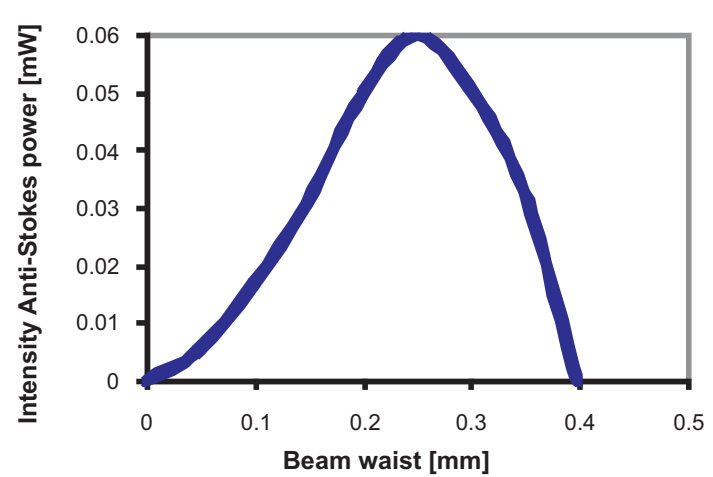

(b)

Fig. 3. Peak power vs beam waist. a) Stokes wave. b) Anti-Stokes waves.

Consider the beam waist is same for all waves and changed $(0.05 \div 0.45) \mu \mathrm{m}$ by outside focusing. The dependence of intra-cavity peak power on beam waist is illustrated in Fig. 3 for two Raman waves. From this figure we can see that: 1) there is an optimal value of beam waist to give maximum value of pulses, which is $0.25 \mu \mathrm{m}$ in this case; 2) in the region of small beam waist $(\leq 0.1 \mu \mathrm{m})$ the peak of pulses is lower; 3$)$ here appears a threshold value of beam waist, which is $0.4 \mu \mathrm{m}$ in this case. This phenomenon can be explained by the spatial distribution of the pump power density overall the fiber.

\section{III.3. Influence of pump energy}

The pump energy is the main factor contributes to stimulated Raman scattering, generally, and to Raman laser power, specially. In nonlinear interaction the pump energy also plays a most important role. Consider pump energy is changed from $0.75 \times 10^{-5} \mathrm{~J}$ to $4.75 \times 10^{-5} \mathrm{~J}$. The dependence of peak power on pump energy is illustrated in Fig. 4 for Stokes and anti-Stokes waves.

From this figure we can see that: 1) there is a threshold value of pump energy, which is about $0.75 \times 10^{-5} \mathrm{~J}$ in this case; 2) there is an optimal value of pump energy, which is to be about $1.75 \times 10^{-5} \mathrm{~J}$ in this case, to give the maximum peak of Stokes and anti-Stokes pulses; 3 ) in the region of low energy the pulse increases with great rate more than one of decreasing in the region of high energy after reaching the maximum. As well known, the Raman gain proportional to the pump power, so the Raman fields increase when the pumping power increases.

After reaches the maximum, with the increasing of pump power the Raman gain is increasing not only at the chosen step (mode) but at other one too, that leads to reduces the Raman power.

\section{CONCLUSION}

The set of dimensionless rate equations of trio-cavity Raman laser pumped by Gaussian beam is derived. Based on this set of equations an optimal value of principle parameters to enhance output power not only for Stokes wave, but also for anti-Stokes one is found out. 


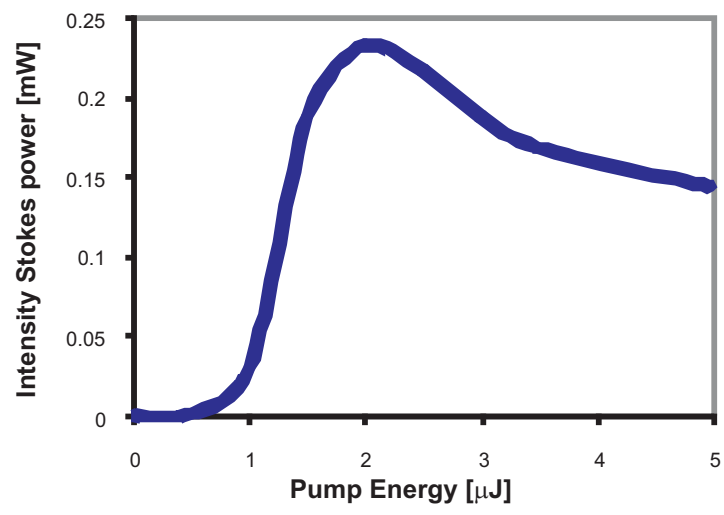

(a)

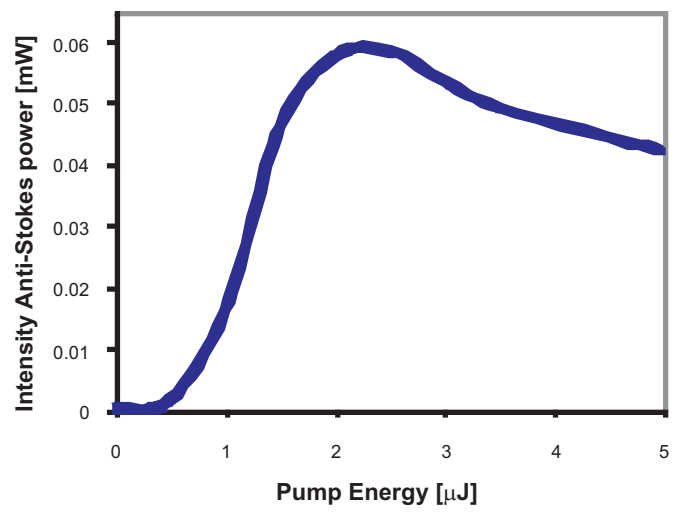

(b)

Fig. 4. Peak power vs pump energy. a) Stokes wave. b) Anti-Stokes wave.

The pulsing process of intra-cavity waves in trio-cavity Raman laser depends on designing parameters as: the pump energy (as so as pump power), beam waist and cavity length (or intra-cavity loss). There are optimal values of above parameters to give maximum peak of pulse (i.e., maximum power) of Stokes and anti-Stokes waves. Moreover, since that the four-wave-mixing appears in this laser the generation and the pulsing process of anti-Stokes always comes together with that of Stokes wave.

\section{REFERENCES}

[1] K. E. Rieckhoff, Appl. Phys. Lett. 9 (1966) 87-98.

[2] J. R. Whinnery, D. T. Miller, and F. Dabby, IEEE J. Quantum Electron. QE-3 (1967) 382-383.

[3] J. K. Brasseur, R. F. Teehan, R. J. Knize, P. A. Roos, and J. L. Carlsten, IEEE J. Quantum Electron QE-37 (2001) 1075-1083.

[4] P. A. Roos, J. K. Brasseur, and J. L. Carlsten, J. Opt. Soc. Am. B17 (2002) 758-763.

[5] J. Bienfang, W. Rudolph, P. A. Roos, L. S. Meng, and J. L. Carlsten, J. Opt. Soc. Am. B19 (2002) $1318-1325$.

[6] P. A.Roos, L. S. Meng, and J. L.Carlsten, J. Opt. Soc. Am. B19 (2002) 1310-1317.

[7] R. Claps et al, Opt. Express 11 (2003) 1731-1739.

[8] T. K. Liang, H. K. Tsang, Appl. Phys. Lett. 84 (2004) 2745-2747.

[9] V. Raghunatham et al, IEICE Electron. Express 12 (2004) 298-304.

[10] T. K. Liang, H. K. Tsang, Proc. Of International Conference on Group IV Photonics, 2004, WA4.

[11] T. Marszalek, "Influence of molecular parameters on dye laser operation," Science thesis, UMK, Torun, Poland. 1974 .

[12] P. A. Roos, L. S. Meng, and J. L. Carlsten, J. Opt. Soc. Am. B19 (2002) $1310-1317$.

Received 27 August 2012. 